\title{
ФОРМУЛЫ ДЛЯ РАСЧЕТА ПРОГИБА И УСИЛИЙ В ШПРЕНГЕЛЬНОЙ ФЕРМЕ С ПРОИЗВОЛЬНЫМ ЧИСЛОМ ПАНЕЛЕЙ
}

\author{
М.Н. Кирсанов \\ Национальный исследовательский университет «МЭИ», г. Москва, РОССИЯ
}

\begin{abstract}
Аннотация: Задача о прогибе плоской статически определимой фермы, имеющей две опоры и сложную решетку, решена в системе компьютерной математики Maple в символьной форме. Использован метод индукции и соответствующие операторы получения и решения рекуррентных уравнений. Найдены выражения для усилий в наиболее сжатых и растянутых стержнях. Проанализировано влияние распределения материала по стержням поясов фермы и решетки, зависимость прогиба от высоты фермы и числа панелей. Получено асимптотическое приближение решения для случая пролета фиксированной длины и заданной нагрузке, равномерно распределенной по узлам нижнего пояса.
\end{abstract}

Ключевые слова: ферма, прогиб, индукция, интеграл Мора, Maple

\section{FORMULAS FOR COMPUTING OF DEFLECTION AND FORCES IN THE TRUSS WITH ARBITRARY NUMBER OF PANELS}

\author{
Mikhail N. Kirsanov \\ National Research University “Moscow Power Engineering Institute”, Moscow, RUSSIA
}

\begin{abstract}
The problem of the deflection of the flat statically determinate truss, having two supports and a complex lattice is solved in the system of computer mathematics Maple in symbolic form. The induction method and special operators receiving and solving recurrence equations are used to obtain the solution. The expression for forces in the most compression and tie rods are detected. Analyzed the distribution of material along the rods of the belts, girders and lattice, dependence of a deflection of the height of the truss and the number of panels. The asymptotic approximation of the solution for the case of fixed span length and a given load, uniformly distributed over the nodes of the lower belt, is obtained.
\end{abstract}

Keywords: truss, deflection, induction, Mohr' integral, Maple

\section{1. ПОСТАНОВКА ЗАДАЧИ}

С развитием систем компьютерной математики (Maple, Mathematica, Maxima и др.) появилась возможность на основе алгоритмов, ранее применяемых в численных расчетах, получать точные формульные решения задач механики, в том числе и механики стержневых систем [1-3]. Ценность аналитического результата, помимо всего прочего, определяется числом независимых параметров исследуемой конструкции. Для ферм это, как правило, размеры, свойство материала и нагрузки. Получить в системе компьютерной мате- матики конечную формулу, включающую в себя эти параметры, не составляет труда. Достаточно уравнения равновесия составить не в численной, а в символьной форме; получить решение системы; и с помощью интеграла Мора вывести итоговую формулу для прогиба, если ставится такая задача. Ввести же в эту формулу число панелей можно далеко не всегда. Во-первых, это возможно при условии регулярности схемы фермы с одним, реже с двумя натуральными числами, характеризующими степень ее сложности. Простая балочная ферма с параллельными поясами и раскосной решеткой - пример такой регу- 
лярной конструкции. Число панелей - это параметр сложности системы. Во-вторых, специфика аналитических преобразований такова, что с увеличением числа стержней, а следовательно, и уравнений равновесия, время преобразований растет значительно быстрее, чем это происходит для численных расчетов, и, начиная с некоторого порядка системы уравнений, время счета настолько велико, что получить аналитическое решение практически невозможно.

Обзор некоторых аналитических решений для плоских ферм содержится в [4-6].

\section{2. ГЕОМЕТРИЯ ФЕРМЫ}

Рассмотрим балочную ферму с решеткой шпренгельного типа (рис. 1). В качестве панели будем считать здесь ячейку периодичности длиной $2 a$ из семи стержней.

В ферме с $n$ панелями в половине пролета содержится

$$
m=16 n+4
$$

стержней, включая три стержня, моделирующие опоры. Число узлов (шарниров) при этом равно $8 n+2$, следовательно, система статически определима. В такой ферме традиционными способами, например, методом сечений, можно вычислить усилия не во всех стержнях. Для некоторых стержней сечений Риттера не существует. Поэтапный метод вырезания узлов возможен, но чтобы добраться до стержней где-нибудь в середине пролета потребуется вырезать много узлов. При этом, как всегда, возникает погрешность вычислений, неизбежная в результате накопления ошибок округления. Поэтому наиболее простым и точным способом решения задачи определения усилий в стержнях остается аналитический метод с использованием метода индукции, который и предлагается применить в данной задаче. Основываясь на алгоритме [7], реализованном на языке Maple (хотя на этом этапе ре- шения задачи вполне допустимы любые другие языки программирования), найдем усилия в стержнях фермы. Введем данные о координатах узлов в программу. Выберем начало координат в левой подвижной опоре. Пронумеруем узлы и стержни (рис. 2).

Имеем следующие координаты узлов:

$$
\begin{aligned}
& x_{i}=a(i-1), y_{i}=0, \quad i=1, \ldots, 4 n+1, \\
& x_{i+4 n+1}=a(2 i-1), \quad y_{i+4 n+1}=h, i=1, \ldots, 2 n, \\
& x_{i+6 n+1}=2 a(i-1), \quad y_{i+6 n+1}=2 h, i=1, \ldots, 2 n+1 .
\end{aligned}
$$

Порядок соединения стержней и узлов зададим по аналогии с заданием вершин и ребер графа условными векторами, координаты которых соответствуют номерам конечных шарниров стержня. Для стержней поясов имеем:

$$
\begin{aligned}
& \left.\bar{V}_{i}=[i, i+1)\right], i=1, \ldots, 4 n, \\
& \bar{V}_{i+4 n}=[i+6 n+1, i+6 n+2], i=1, \ldots, 2 n, \\
& \bar{V}_{i+6 n}=[2 i-1, i+6 n+1], i=1, \ldots, 2 n+1,
\end{aligned}
$$

Стержни решетки заданы векторами:

$$
\begin{aligned}
\bar{V}_{i+8 n+1}=[2 i, i+4 n+1], i=1, \ldots, 2 n, \\
\left.\bar{V}_{i+10 n+1}=[2 i-1, i+4 n+1)\right], \\
\bar{V}_{i+11 n+1}=[2 i+2 n+1, \quad i+5 n+1], \\
\left.\bar{V}_{i+12 n+1}=[i+6 n+1, i+4 n+1)\right], \\
\bar{V}_{i+13 n+1}=[i+7 n+2, i+5 n+1], \\
\left.\bar{V}_{i+14 n+1}=[i+6 n+2, i+4 n+1)\right], \\
\bar{V}_{i+15 n+1}=[i+7 n+1, \quad i+5 n+1], i=1, \ldots, n .
\end{aligned}
$$

Для вычисления направляющих косинусов, являющихся коэффициентами уравнений равновесия узлов в проекциях, вычислим длины стержней и проекции их условных векторных представлений на оси координат:

$$
\begin{aligned}
& l_{i}=\sqrt{l_{1, i}{ }^{2}+l_{2, i}{ }^{2}}, l_{1, i}=x_{V_{2, i}}-x_{V_{1, i}}, \\
& l_{2, i}=y_{V_{2, i}}-y_{V_{1, i}}, \quad i=1, \ldots, m .
\end{aligned}
$$




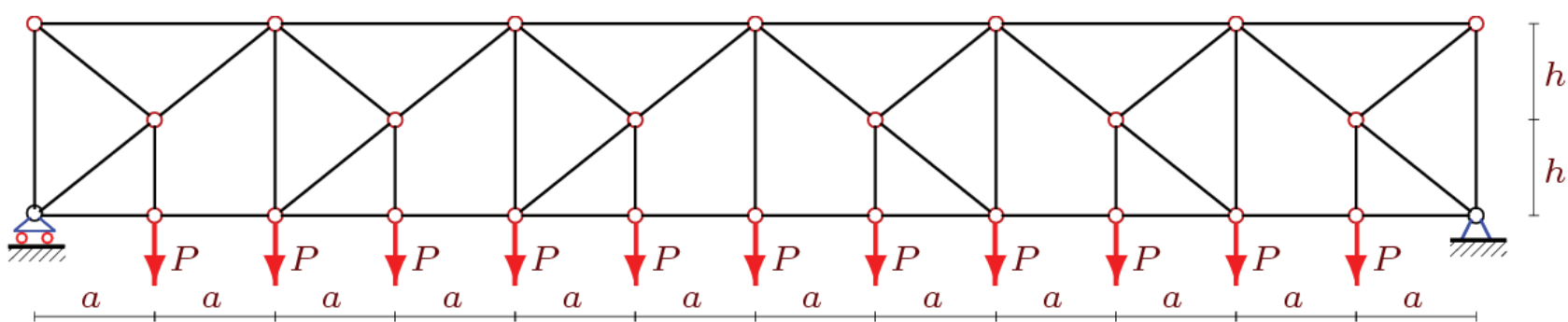

Рисунок 1. Ферма, $n=3$.

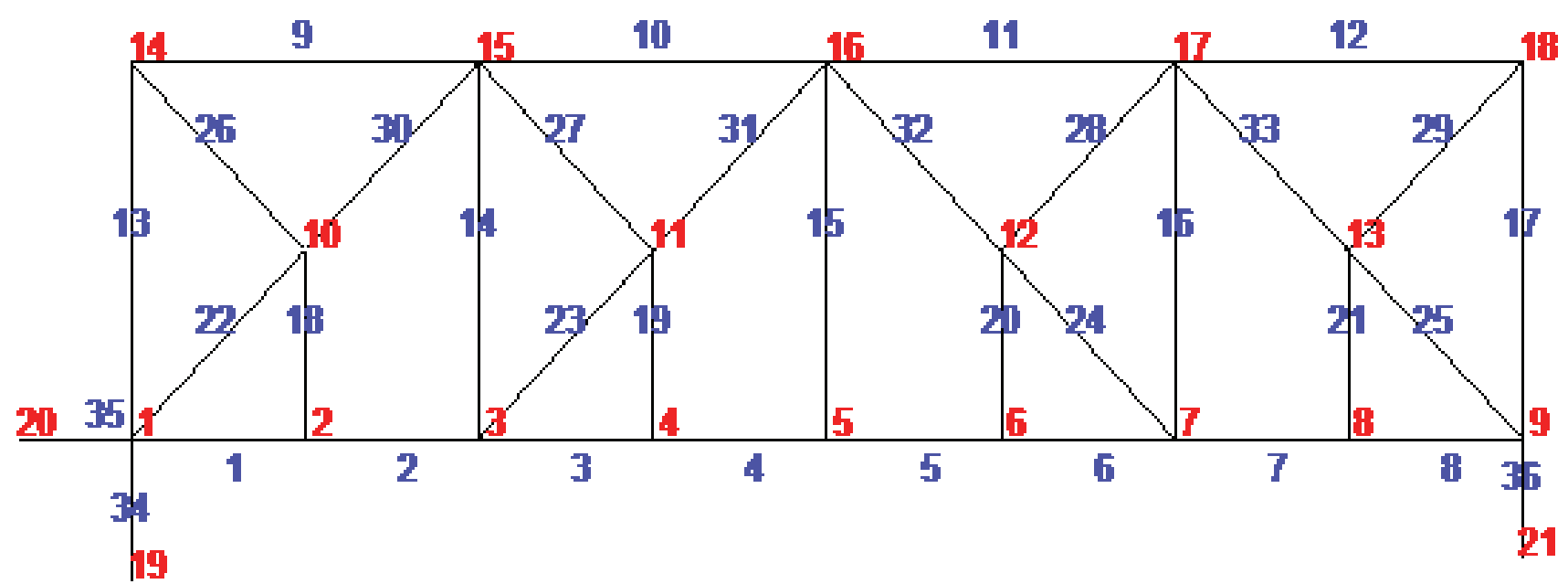

Рисунок 2. Номера стержней и узлов, $n=2$.

В номере $V_{j, i}$ индекс $j$ принимает значения 1 или 2 и соответствует номеру компоненты вектора $\bar{V}_{i}$, второй - номеру стержня. Матрица направляющих косинусов имеет следующие элементы:

$$
\begin{gathered}
G_{k, i}=-l_{j, i} / l_{i}, \quad k=2 V_{i, 2}-2+j, k \leq n_{s}, \\
j=1,2, \quad G_{k, i}=l_{j, i} / l_{i}, k=2 V_{i, 1}-2+j, \\
k \leq m, \quad j=1,2, \quad i=1, \ldots, m .
\end{gathered}
$$

Система уравнений равновесия узлов имеет вид

$$
\mathbf{G} \bar{S}=\bar{B},
$$

где $\bar{S}=\left\{S_{1}, \ldots, S_{m}\right\}-$ вектор усилий, $\bar{B}-$ вектор внешних нагрузок. Для определения прогиба фермы используем интеграл Мора,

$$
\Delta=P \sum_{i=1}^{m-3} S_{i}^{(P)} S_{i}^{(1)} l_{i} /\left(E F_{i}\right),
$$

где суммирование ведется только по стержням фермы и обозначено: $S_{i}^{(1)}-$ усилия от единичной силы, приложенной к середине нижнего пояса (узел $2 n+1$, рис. 2), $S_{i}^{(P)}-$ усилия в стержнях от заданной нагрузки, $l_{i}$ - длины стержней. Жесткости стержней $E F_{i}$ в общем случае разные. Пусть площади сечений верхнего и нижнего пояса выражаются через некоторую условную (можно и единичную) площадь: $F_{i}=F / \gamma_{1}, i=1, \ldots, 6 n$. Площади сечений всех стержней решетки имеют вид: $F_{i}=F / \gamma_{2}, i=6 n+1, \ldots, m-3$. Последовательный аналитический расчет ферм с увеличивающимся всякий раз числом панелей выявил, что формула для прогиба имеет один и тот же вид:

$$
\Delta=P \frac{A_{n} a^{3} \gamma_{1}+\left(H_{n} h^{3}+C_{n} c^{3}\right) \gamma_{2}}{2 h^{2} E F}
$$

отличаясь только коэффициентами при степенях (кубах) размеров $a, h$ и 


$$
c=\sqrt{a^{2}+h^{2}} .
$$

Для того, чтобы найти общий член последовательности коэффициентов 4, 58, 282, 876, 2120, 4374, 8078, 13752, 21996, 33490 при $a^{3}$ необходимо с помощью специального оператора rgf_findrecur найти рекуррентное уравнение, которому удовлетворяет эта последовательность

$$
A_{n}=5 A_{n-1}-10 A_{n-2}+10 A_{n-3}-5 A_{n-4}+A_{n-5},
$$

а затем решить его, используя оператор rsolve. Решение имеет вид

$$
A_{n}=n\left(10 n^{3}+5 n-3\right) / 3 .
$$

Аналогично получаем выражения для других коэффициентов:

$$
H_{n}=4\left(n^{2}-n+1\right), C_{n}=n(4 n-1) .
$$

\section{3. АНАЛИЗ ПРОГИБА}

Рассмотрим случай фермы с заданным пролетом $L$ и произвольным числом панелей, так что

$$
a=L /(4 n) .
$$

Аналитическая форма решения позволяет наглядно выявить некоторые его особенности. Зафиксируем также общую нагрузку на ферму

$$
P_{\text {sum }}=(4 n-1) P \text {. }
$$

Введем обозначение для безразмерного прогиба

$$
\Delta^{\prime}=\Delta E F /\left(P_{\text {sum }} L\right)
$$

На рисунке 3 при $\gamma_{1}=\gamma_{2}=1$ и $L=50$ м даны кривые зависимости прогиба (1) от числа панелей, показывающие, что в данной постановке решение имеет явно выраженный экстремум, зависящий от высоты фермы. Это дает возможность проектировщику выбрать оптимальное число панелей. Кроме того заметны и асимптоты кривых. Для аналитического их выражения $\Delta^{\prime}=\Delta_{0}+k n$ вычислим пределы:

$$
\begin{gathered}
k=\lim _{n \rightarrow \infty} \Delta^{\prime} / n=\gamma_{2} h / L, \\
\Delta_{0}=\lim _{n \rightarrow \infty}\left(\Delta^{\prime}-k n\right)=\left(5 \gamma_{1} L^{3}-288 \gamma_{2} h^{3}\right) /\left(768 L h^{2}\right) .
\end{gathered}
$$

Интересно отметить, что угол наклона асимптоты зависит только от жесткости решетки $\gamma_{2}$, высоты $h$ и пролета $L$. Зависимость прогиба от высоты фермы (рис.4) также обнаруживает экстремум. С увеличением жесткости решетки (уменьшение $\gamma_{2}$ ) оптимальная по жесткости высота фермы увеличивается.

\section{4. УСИЛИЯ В СТЕРЖНЯХ}

Для определения закономерности образования коэффициентов в усилиях стержней потребовалась последовательность расчетов меньшей длины. Индукция по результатам шести ферм с числом панелей от 1 до 6 дает общую формулу для наиболее растянутого стержня в середине нижнего пояса

$$
S_{2 n}^{(P)}=P n^{2} a / h
$$

Аналогично, формула для усилия в наиболее сжатом стержне середины верхнего пояса имеет вид:

$$
S_{5 n}^{(P)}=-P\left(2 n^{2}-1\right) a /(2 h) .
$$




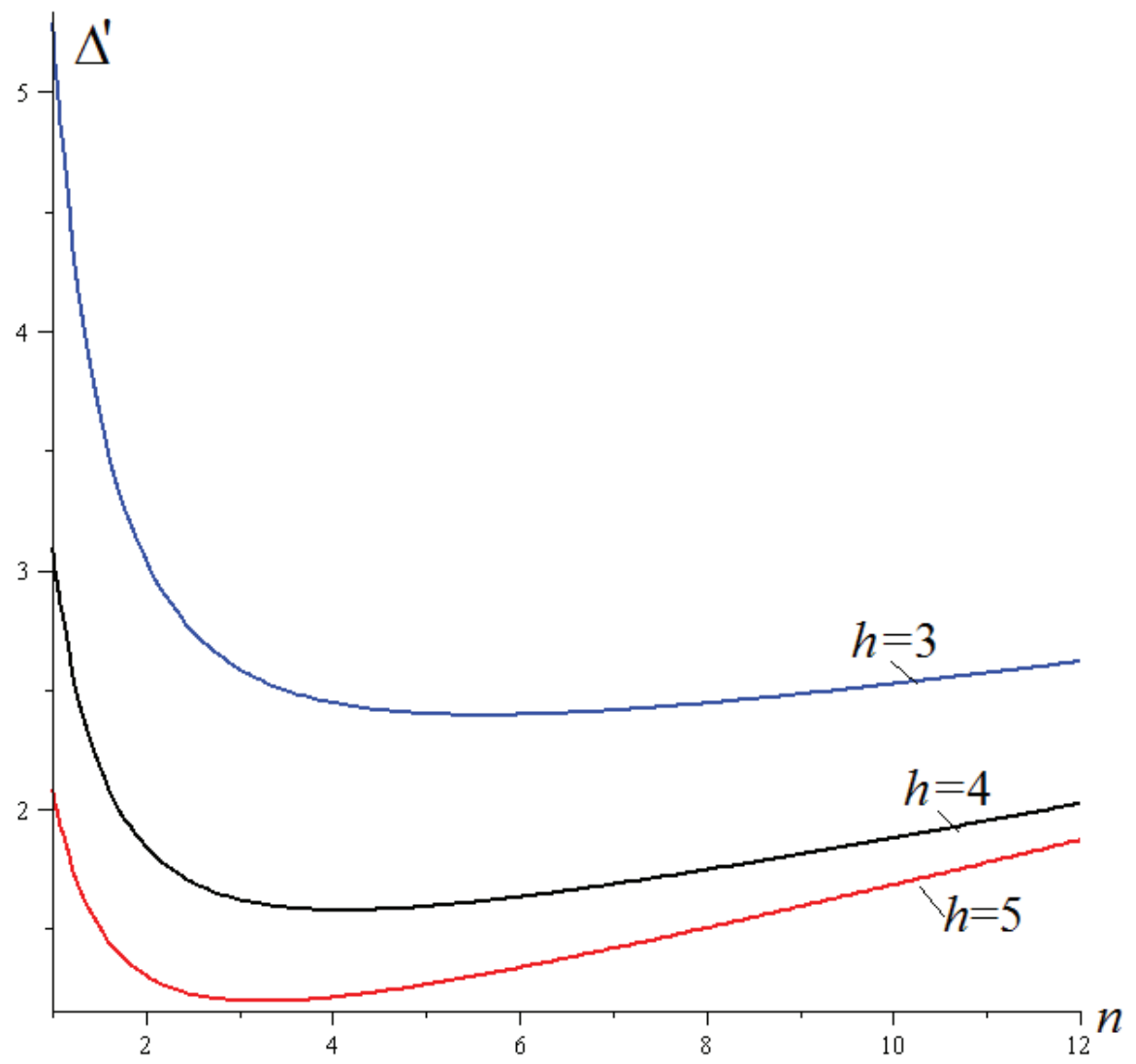

Рисунок 3. Зависимость прогиба от числа панелей.

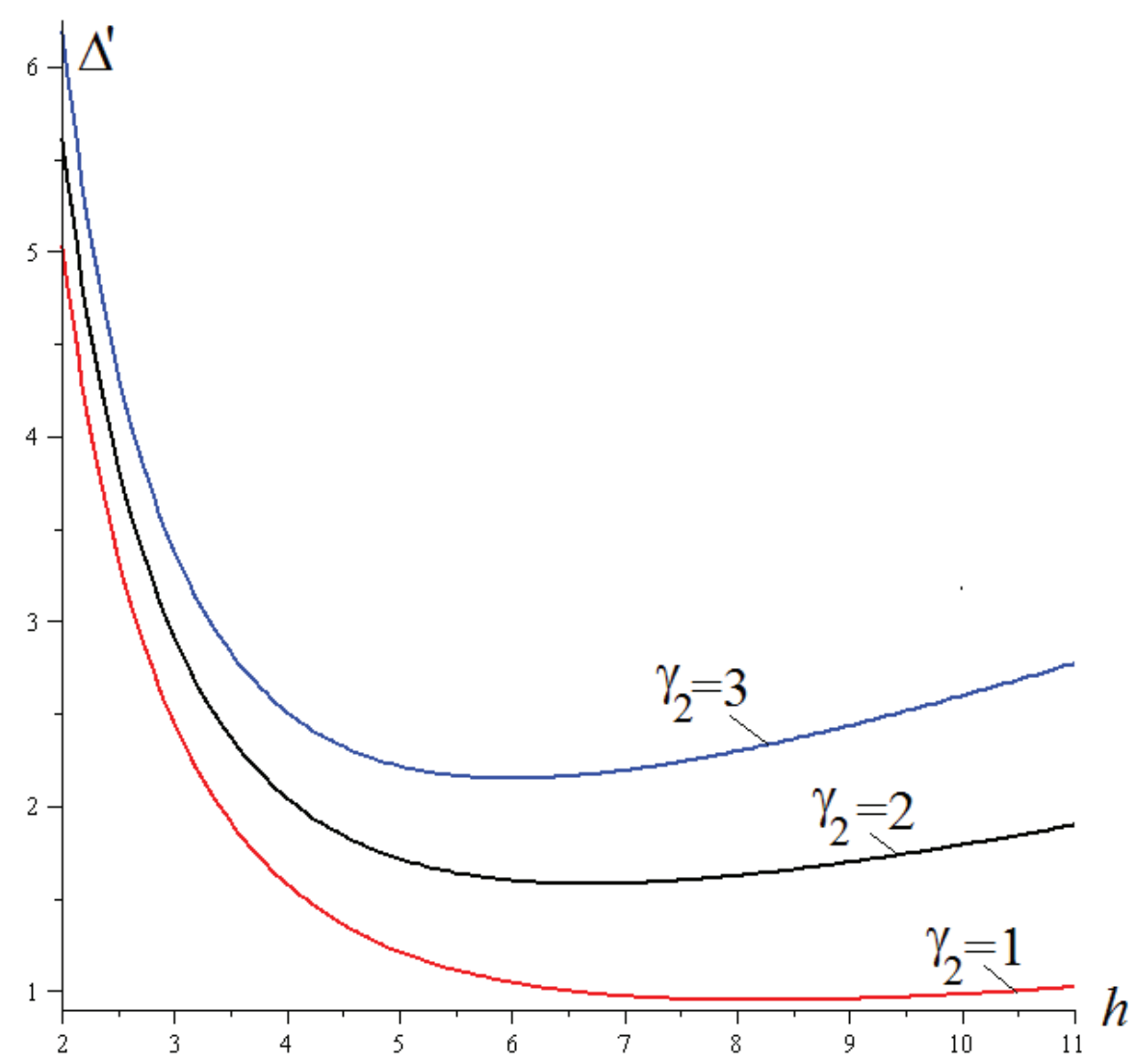

Рисунок 4. Зависимость прогиба от высоть фермыл. 


\section{СПИСОК ЛИТЕРАТУРЫ}

1. Kirsanov M. N. Stress State and Deformation of a Rectangular Spatial Rod Cover// Scientific Herald of the Voronezh State University of Architecture and Civil Engineering. Construction and Architecture. 2016. N 3 (31). Pp. 71-79.

2. Кирсанов М.Н. Аналитический расчет балочной фермы с решеткой типа "butterfly"// Строительная механика и расчет сооружений. 2016. № 4. С. 2-5.

3. Al-Shahrabi A.M., Kirsanov M.N. Line of influence of the deflection for cantilever truss //Вестник научных конференций. 2016. № 2-1(6). С. 6-7.

4. Тиньков Д.В. Анализ точных решений прогиба регулярных шарнирностержневых конструкций// Строительная механика инженерных конструкций и сооружений. 2015. №6. С. 21-28.

5. Тиньков Д.В. Сравнительный анализ аналитических решений задачи о прогибе ферменных конструкций // Инженерно-строительный журнал. 2015. №5(57). C. 66-73.

6. Кийко Л.К. Аналитическая оценка прогиба арочной фермы под действием ветровой нагрузки // Научный вестник. 2016. № 1 (7). С. 247-254.

7. Кирсанов М.Н. Maple и Maplet. Решения задач механики. СПб.: Изд-во Лань, 2012. 512 c.

\section{REFERENCES}

1. Kirsanov M.N. Stress State and Deformation of a Rectangular Spatial Rod Cover. // Scientific Herald of the Voronezh State University of Architecture and Civil Engineering. Construction and Architecture, 2016, No. 3(31), pp. 71-79.

2. Kirsanov M.N. Analiticheskij Raschet Balochnoj Fermy s Reshetkoj Tipa "Butterfly" [An Analytical Analysis of a Beam Farm with a "Butterfly"]. // Stroitel'naya
Mekhanika i Raschet Sooruzhenij, 2016, No. 4, pp. 2-5.

3. Al-Shahrabi A. M., Kirsanov M.N. Line of Influence of the Deflection for Cantilever Truss. // Vestnik nauchnyh konferencij, 2016, No. 2-1(6), pp. 6-7.

4. Tinkov D.V. Analiz Tochnyh Reshenij Progiba Regulyarnyh SharnirnoSterzhnevyh Konstrukcij [Analysis of Exact Solutions to the Deflection of Regular Hinge-Rod Structures]. // Stroitel'naya Mekhanika Inzhenernyh Konstrukcij i Sooruzhenij, 2015, No. 6, pp. 21-28.

5. Tinkov D.V. Sravnitel'nyj Analiz Analiticheskih Reshenij Zadachi o Progibe Fermennyh Konstrukcij [Comparative Analysis of Analytical Solutions of the Problem of Deflection of Truss Structures]. // Inzhenerno-Stroitel'nyj Zhurnal, 2015, No. 5(57), pp. 66-73.

6. Kijko L.K. Analiticheskaya Otsenka Progiba Arochnoj Fermy pod Dejstviem Vet-rovoj Nagruzki [Analytical Estimation of Arch Deflection Under the Influence of Wind Load]. // Nauchnyj vestnik, 2016, No. 1(7), pp. 247-254.

7. Kirsanov M.N. Maple i Maplet. Resheniya Zadach Mekhaniki [Maple and Maplet. Solution of Problems of Structural Analysis]. Saint-Petersburg, Lan, 2012, 512 pages.

Кирсанов Михаил Николаевич, профессор, доктор физико-математических наук, Национальный исследовательский университет «МЭИ», кафедра теоретической механики и мехатроники; 111250, Россия, г. Москва, ул. Красноказарменная, дом 14; тел. +7(495)362-73-14, e-mail: c216@ya.ru.

Kirsanov Mikhail Nikolaevich, Professor, Doctor of Physico-Mathematical Sciences, National Research University "Moscow Power Engineering Institute", Department of Theoretical Mechanics and Mechatronics; 111250, Russia, Moscow, Krasnokazarmennaya str., 14; phone+7(495)362-73-14, e-mail: c216@ya.ru. 